\title{
Measurement of epidermal thickness in a patient with psoriasis by computer-supported image analysis
}

M. Alper ${ }^{1}$, A. Kavak², A.H. Parlak², R. Demirci ${ }^{4}$,

I. Belenli ${ }^{4}$ and N. Yesildal ${ }^{3}$

\author{
Departments of ${ }^{1}$ Pathology, ${ }^{2}$ Dermatology, and ${ }^{3}$ Public Health, \\ Faculty of Medicine, and \\ ${ }^{4}$ Technical Education Faculty, University of Abant Izzet Baysal, \\ Konuralp, Düzce, Turkey
}

Correspondence

M. Alper

Aibü Düzce Tip Fakültesi

Patoloji Bölümü

Konuralp, Düzce

Turkey

Fax: +90-380-541-4213

E-mail: muratalper@tusdata.com

Received March 17, 2003

Accepted September 19, 2003

\begin{abstract}
The aim of the present study was to measure full epidermal thickness, stratum corneum thickness, rete length, dermal papilla widening and suprapapillary epidermal thickness in psoriasis patients using a light microscope and computer-supported image analysis. The data obtained were analyzed in terms of patient age, type of psoriasis, total body surface area involvement, scalp and nail involvement, duration of psoriasis, and family history of the disease. The study was conducted on 64 patients and 57 controls whose skin biopsies were examined by light microscopy. The acquired microscopic images were transferred to a computer and measurements were made using image analysis. The skin biopsies, taken from different body areas, were examined for different parameters such as epidermal, corneal and suprapapillary epidermal thickness. The most prominent increase in thickness was detected in the palmar region. Corneal thickness was more pronounced in patients with scalp involvement than in patients without scalp involvement $(\mathrm{t}=-2.651, \mathrm{P}=0.008)$. The most prominent increase in rete length was observed in the knees (median: $491 \mu \mathrm{m}$, $\mathrm{t}=10.117, \mathrm{P}=0.000$ ). The difference in rete length between patients with a positive and a negative family history was significant $(\mathrm{t}=$ $-3.334, \mathrm{P}=0.03$ ), being $27 \%$ greater in psoriasis patients without a family history. The differences in dermal papilla distances among patients were very small. We conclude that microscope-supported thickness measurements provide objective results.
\end{abstract}

\section{Introduction}

Psoriasis is a noninfectious, inflammatory, and hyperproliferative skin disease. Histologically, fully developed psoriasis lesions are characterized by acanthosis with regular elongation of rete ridges, thickening in their lower portion, thinning of the suprapapillary epidermis, reduced to absent granu-
Key words

- Psoriasis

- Epidermis

- Thickness

- Computer-supported

image analysis lar layer confluent parakeratosis, presence of Munro microabscess, elongation and edema of the dermal papillae, and dilated and tortuous capillaries. Many methods have been used to estimate remission and exacerbation periods on the basis of clinical findings and some objective criteria, such as ultrasound, reflectance colorimetry, computerized video image analysis, pseudo-elongation of capil- 
laries, evaluation of skin surface hydration, and nitric oxide production (1-3).

Our objective was to perform the following sensitive measurements of epidermal parameters in psoriasis patients: stratum corneum thickness, rete length, epidermal thickness, suprapapillary epidermal thickness, and dermal papilla distances by computer-supported image analysis and to correlate them with properties such as type of psoriasis, percent of total body surface area (\%TBSA) involvement, scalp and nail involvement, and a family history of psoriasis.

\section{Material and Methods}

One hundred and twenty-one skin biopsies performed at the Abant Izzet Baysal University Medical Faculty, Düzce, Turkey between January 1999 and June 2002 were examined. This series consisted of 64 patients with a clinical and histopathological diagnosis of psoriasis and 57 control subjects. Psoriasis patients with active dermatological complaints who had come to the Dermatology Clinic were screened and those who fulfilled the inclusion criteria were included in the study. Inclusion criteria were: psoriasis patients who had not received systemic steroids within the last 3 months, patients who had not used topical steroids, calcipotriol or moisturizing agents within the last 6 months, and patients who had not received psoralen + ultraviolet $\mathrm{A}$ treatment within the last 6 months. The control group was selected from our archives and consisted of patients with unexpected epidermal hyperplasia and hypoplasia, lesions affecting deep regions of the skin (panniculitis, small cystic lesions located in the deep dermis, etc.), and mild lesions. Age, sex, disease duration, extent of disease, family history, nail involvement, and scalp involvement were determined and evaluated by the same dermatologist. Biopsies were performed after informed consent was obtained from the patients or their parents. The study was evaluated and approved by the Ethics Committee of Düzce Medical Faculty.

Histopathological images of hematoxylin-eosin-stained vertical 5- $\mu \mathrm{m}$ thick crosssections taken with an Olympus BX50 microscope were transferred to a computer with a Panasonic GP-KR222 camera and converted to BMP files. A PathPic image analysis software developed at the Abant Izzet Baysal University Technical Education Faculty was used to examine the images and perform the measurements. Full epidermal thickness, stratum corneum thickness, rete length, dermal papilla distances, and suprapapillary epidermal thickness were measured. First, the distance between the top and the bottom of the rete was measured and full epidermal thickness was determined. Next, the stratum corneum (from the granular layer to the tip), rete (from the upper part of the granular layer to the bottom of the epidermis), suprapapillary epidermis (from the dermal papilla to the top of the granular layer), and dermal papillae (the area where vessels are dilated beneath the epidermis) were measured. For each biopsy specimen, five different areas representing the lesion were measured and the average was calculated. Psoriasis patients with a positive family history, nail involvement and scalp involvement were compared to patients without these conditions and to the control group.

The psoriasis patient group consisted of 32 females and 32 males, and the control group consisted of 28 females and 29 males.

Disease types were classified as plaque, palmar, guttate, erythrodermic, and inverse and were detected at the following rates in psoriasis patients: plaque type in 54 (84.3\%), palmar type in $4(6.2 \%)$, guttate type in 4 $(6.2 \%)$, erythrodermic type in $1(1.5 \%)$, and inverse type in $1(1.5 \%)$.

The biopsies were taken from the knees in $22(34.4 \%)$ patients, from the palmar region in $13(20.3 \%)$, from the elbows in 11 (17.2\%), from the legs in $10(15.6 \%)$, and from the trunk in $8(12.5 \%)$. For the control 
group, the biopsies were taken from the knees in $16(28.1 \%)$ cases, from the palmar region in $15(26.3 \%)$, from the legs in $10(17.5 \%)$, from the elbows in $9(15.8 \%)$, and from the trunk in $7(12.3 \%)$.

The subjects were divided into three age groups: 2-34 years, 35-54 years, and 55 years or more. Patient age ranged from 2 to 95 years (mean: $39 \pm 2.09$ years) and control subject age ranged from 10 to 69 years (mean: $37.8 \pm 1.89$ years). The 2 - to 34 -year age group consisted of 25 patients and 20 controls, the 35- to 54-year age group of 29 patients and 31 controls, and the above 55year age group of 10 patients and 6 controls.

$\%$ TBSA was calculated according to the rule of nines and was divided into two groups: $1-5 \%$ and $6 \%$ and above. \%TBSA ranged from 1 to $80 \%$ (median: 9.93\%), with 36 cases in the first group and 28 cases in the second.

Disease duration ranged from 1 to 420 months (mean: $51 \pm 11$ months) and was divided into two groups: 1-59 and 60 months or more, with 33 cases in the first group and 31 cases in the second group.
The relationships between these groups and the control group were examined. The biopsy types and sites of the case groups were compared with each other and with the control group.

Data were analyzed statistically using the SPSS statistical program. The Student $t$-test was used to compare the averages of continuous variables. When parametric assumptions could not be satisfied, the MannWhitney U-test was used.

\section{Results}

The epidermal measurements for the case and control groups are presented in Table 1. Compared to control, the full epidermal thickness of psoriasis patients was 2.71 times greater $(\mathrm{t}=10.45, \mathrm{P}=0.000)$, corneal thickness was 2.41 times greater $(\mathrm{t}=4.15, \mathrm{P}=$ $0.000)$ and rete length was 2.47 times greater $(\mathrm{t}=12.95, \mathrm{P}=0.000)$. Suprapapillary epidermal thickness was similar for the case and control groups $(\mathrm{t}=0.67, \mathrm{P}=0.503)$. However, dermal papillae were $13 \%$ narrower in the case group than in the control group $(\mathrm{t}=$

Table 1. Epidermal thickness measurements of psoriasis patients and controls.

\begin{tabular}{lccccc}
\hline & $\begin{array}{c}\text { Full epidermal } \\
\text { thickness }(\mu \mathrm{m})\end{array}$ & $\begin{array}{c}\text { Stratum } \\
\text { corneum } \\
\text { thickness }(\mu \mathrm{m})\end{array}$ & $\begin{array}{c}\text { Rete length } \\
(\mu \mathrm{m})\end{array}$ & $\begin{array}{c}\text { Suprapapillary } \\
\text { epidermal } \\
\text { thickness }(\mu \mathrm{m})\end{array}$ & $\begin{array}{c}\text { Dermal papilla } \\
\text { distance }(\mu \mathrm{m})\end{array}$ \\
\hline Control (57) & 212 & 40 & 174 & 64 & 50 \\
Patients (64) & 576 & 98 & 431 & 65 & 44 \\
(+) Family history (8) & 460 & 137 & 358 & 70 & 39 \\
(-) Family history (56) & 588 & 96 & 455 & 65 & 45 \\
(+) Scalp involvement (27) & 567 & 81 & 441 & 66 & 45 \\
(-) Scalp involvement (37) & 594 & 142 & 418 & 64 & 40 \\
(+) Nail involvement (11) & 567 & 81 & 441 & 73 & 45 \\
(-) Nail involvement (53) & 586 & 102 & 426 & 71 & 42 \\
Disease duration group 1 (33) & 590 & 166 & 424 & 64 & 51 \\
Disease duration group 2 (31) & 540 & 81 & 407 & 78 & 36 \\
\%TBSA group 1 (36) & 621 & 119 & 441 & 57 & 43 \\
\%TBSA group 2 (28) & 558 & 91 & 407 & 68 & 44 \\
Plaque (54) & 576 & 96 & 438 & 63 & 47 \\
Palmar disease (4) & 719 & 329 & 480 & 101 & 29 \\
Guttate disease (4) & 361 & 64 & 286 & 47 & 46 \\
\hline
\end{tabular}

The number of subjects in each group is given in parentheses. The data are reported as medians in micrometers. \%TBSA $=$ percent of total body surface area involvement; $(+),(-)=$ positive and negative, respectively. 
$-2.42, \mathrm{P}=0.018)$. The difference in rete length between patients having a positive and a negative family history was significant $(\mathrm{t}=-3.334, \mathrm{P}=0.03)$, being $27 \%$ greater in psoriasis patients without a family history. The differences in the thickness of epidermis, stratum corneum and suprapapillary epidermis, and also in dermal papilla distance between case and control groups were not statistically significant $(\mathrm{P}>0.05)$.

The epidermal measurements according to type of psoriasis are given in Table 1 . When the measurements for the patients with plaque type psoriasis were compared with those for the control group, there were significant differences in epidermal thickness $(\mathrm{t}=10.471, \mathrm{P}=0.000)$, corneal thickness $(\mathrm{t}=3.819, \mathrm{P}=0.000)$, rete length $(\mathrm{t}=12.111$, $\mathrm{P}=0.000)$, and dermal papilla distance $(\mathrm{t}=$ $-1.977, \mathrm{P}=0.042$ ) but not in suprapapillary epidermal thickness $(t=0.652, P=0.516)$.

The results concerning the biopsy location in patients and controls are presented in Table 2. When the measurements in the elbows of patients and controls were compared, the differences in epidermal thickness $(t=3.564, P=0.002)$ and rete length $(\mathrm{t}=5.588, \mathrm{P}=0.000)$ were statistically significant while there was no significant difference in stratum corneum $(\mathrm{t}=1.348, \mathrm{P}=$
0.194), suprapapillary epidermal thickness $(\mathrm{t}=-0.468, \mathrm{P}=0.645)$, or dermal papilla distances $(\mathrm{t}=-1.021, \mathrm{P}=0.321)$. Again, when the lesion areas measured in the knees of the patients and the control group were compared, the differences in epidermal thickness $(t=7.113, P=0.000)$ and rete length $(\mathrm{t}=10.117, \mathrm{P}=0.000)$ were statistically significant, whereas no significant differences in stratum corneum $(\mathrm{t}=1.494, \mathrm{P}=$ $0.144)$, suprapapillary epidermal thickness $(\mathrm{t}=0.518, \mathrm{P}=0.608)$, or dermal papilla distances $(t=-1.170, P=0.250)$ were $o b$ served. When the lesion areas measured in the legs of the patients and the control group were compared, the differences in epidermal thickness $(t=6.002, P=0.000)$, rete length $(\mathrm{t}=6.187, \mathrm{P}=0.000)$, and stratum corneum thickness $(\mathrm{t}=2.438, \mathrm{P}=0.018)$ were statistically significant, whereas no significant difference in suprapapillary epidermal thickness $(t=-0.433, P=0.670)$ or dermal papilla distance $(\mathrm{t}=-0.439, \mathrm{P}=0.666)$ was observed. In the trunk, when the results for patients and controls were compared, the differences in epidermal thickness $(\mathrm{t}=3.322$, $\mathrm{P}=0.006)$, rete length $(\mathrm{t}=3.002, \mathrm{P}=0.010)$, stratum corneum thickness $(\mathrm{t}=2.438, \mathrm{P}=$ $0.030)$, and dermal papilla distances $(\mathrm{t}=$ $2.544, \mathrm{P}=0.024)$ were statistically signifi-

\begin{tabular}{lccccc}
\multicolumn{6}{l}{ Table 2. Epidermal thickness measurements of psoriasis patients and controls according to biopsy location. } \\
\hline Biopsy location & $\begin{array}{c}\text { Full epidermal } \\
\text { thickness }(\mu \mathrm{m})\end{array}$ & $\begin{array}{c}\text { Stratum } \\
\text { corneum } \\
\text { thickness }(\mu \mathrm{m})\end{array}$ & $\begin{array}{c}\text { Rete length } \\
(\mu \mathrm{m})\end{array}$ & $\begin{array}{c}\text { Suprapapillary } \\
\text { epidermal } \\
\text { thickness }(\mu \mathrm{m})\end{array}$ & $\begin{array}{c}\text { Dermal papilla } \\
\text { distance }(\mu \mathrm{m})\end{array}$ \\
\hline Patient elbow (11) & 500 & 133 & 361 & 60 & 51 \\
Control elbow (9) & 326 & 73 & 225 & 88 & 69 \\
Patient knee (22) & 610 & 87 & 491 & 72 & 40 \\
Control knee (16) & 208 & 36 & 157 & 65 & 37 \\
Patient leg (10) & 532 & 91 & 447 & 53 & 56 \\
Control leg (10) & 195 & 37 & 159 & 69 & 45 \\
Patient trunk (8) & 365 & 64 & 307 & 53 & 46 \\
Control trunk (7) & 204 & 23 & 180 & 40 & 63 \\
Patient palmar region (13) & 663 & 192 & 388 & 78 & 35 \\
Control palmar region (15) & 207 & 45 & 162 & 43 & 46
\end{tabular}

The number of subjects in each group is given in parentheses. The data are reported as medians in micrometers. 
cant, whereas there was no significant difference in suprapapillary epidermal thickness $(t=1.066, P=0.306)$. When the results for patients and controls were compared for the palmar region, the differences in epithelial thickness $(t=4.104, P=0.001)$, rete length $(\mathrm{t}=4.616, \mathrm{P}=0.000)$ and stratum corneum thickness $(\mathrm{t}=3.128, \mathrm{P}=0.004)$ were statistically significant, whereas there were no significant differences in suprapapillary epidermal thickness $(\mathrm{t}=1.014, \mathrm{P}=$ $0.320)$ or dermal papilla distance $(t=-1.526$, $\mathrm{P}=0.139$ ).

A significant difference in corneal thickness of knee lesions was observed between age groups, with thickness increasing with age. Similarly, rete length also increased with age $(\mathrm{P}=0.028$, chi square $=3.11)$. A significant difference for suprapapillary epidermal thickness between the patients and control groups was not determined. There was no significant correlation between dermal papilla distances and age groups $(\mathrm{P}>$ $0.05)$. No significant difference in epithelial thickness was observed in palmar regions, elbows, legs and trunk of the various age groups compared to control ( $\mathrm{P}>0.05)$.

A family history of psoriasis was positive in $8(12.5 \%)$ patients and negative in 56 $(87.5 \%)$. Scalp involvement was present in $27(42.2 \%)$ cases and absent in $37(57.8 \%)$. Also, nail involvement was present in 11 (17.2\%) cases and absent in 53 (82.8\%). Measurements of epidermal thickness according to \% TBSA are presented in Table 1. The difference in epithelial thickness between patients with and without a family history was not significant $(\mathrm{P}>0.05)$.

No significant correlation between epidermal thickness and duration of the disease was found $(\mathrm{P}>0.05$; Table 1$)$.

Corneal thickness was significantly greater in psoriasis patients with scalp involvement $(\mathrm{t}=-2.651, \mathrm{P}=0.008)$. The differences in full epidermal thickness, rete length, suprapapillary epidermal thickness, and dermal papilla distance between these groups were not statistically significant $(\mathrm{P}>$ $0.05)$. In addition, there was no significant difference in epithelial thickness between psoriasis patients with and without nail involvement $(\mathrm{P}>0.05)$.

\section{Discussion}

Many studies have been conducted to estimate the remission and exacerbation of psoriasis on the basis of clinical findings and objective parameters. Many methods such as ultrasound, reflectance colorimetry, computerized video image analysis, pseudo-elongation of capillaries, evaluation of skin surface hydration, changes in skin blood flow, and nitric oxide production have been investigated for this purpose (1-7). The aim of all of these studies was to determine objective parameters for the definition of patient involvement by psoriasis, a disease that negatively affects the quality of life and psychology of the patient. The disease requires long and relatively expensive treatment and methods are needed to contribute to its diagnosis and to estimate periods of remission and exacerbation. In these studies, inter- and intraobserver variations must be minimized and the least invasive methods should be used. The clinical relevance of the findings must be clear and reliable. Many studies have been conducted thus far, but their practical usefulness has not been demonstrated. Most of them have determined that psoriasis area and severity index should be taken into account as part of the clinical criteria concerning erythema and plaque thickness (1$3)$. Ormerod et al. (1) compared subjective measures such as erythema, elevation, and scaling separately and as a whole with objective criteria such as ultrasound, reflectance colorimetry, computerized video image analysis, and nitric oxide production in their series of 12 patients and concluded that nitric oxide production was the most powerful objective measure.

Bacharach-Buhles et al. (2) investigated 
intrapapillary vessels in active and healing plaques by computer-supported image analysis in a series of 120 cases. They reported that the vessels were not proliferative, and that there was no difference in the arrangement of the vessels between the psoriatic lesion and the adjacent non-involved skin during separate periods.

Ashcroft et al. (5) examined the status of clinical outcome measures used in psoriasis research. The measures most commonly used were individual sign scores, e.g., for erythema, plaque thickness or scaling, and pooled indices, e.g., psoriasis area and severity index. Similarly, Ring et al. (4) also studied psoriasis area and severity index.

In the series of 70 cases reported by Kim et al. (3), erythema, scaling and infiltration of individual lesions were assessed subjectively by determining the extent of involvement. The functional status of the stratum corneum was evaluated by measuring electrical capacitance and conductance of the involved and uninvolved skin of psoriatic patients, and transepidermal water loss was measured. The authors showed that psoriatic attacks were associated with dry skin.

Tanaka et al. (6) reported that computerassisted area measurement of skin lesions was very useful in evaluating the severity of the disease. However, they did not measure epithelial thickness.

In contrast with other studies, we concentrated on sensitive measurements of epithelial thickness. We performed sensitive measurements of epidermal thickness such as stratum corneum thickness, rete length, suprapapillary epidermal thickness, and dermal papilla distances using a microscope and a computer. The relationship between these measured properties and the clinical findings, such as type of psoriasis, \% TBSA, scalp involvement, nail involvement, and the presence of a family history was then investigated.

The results showed that full epidermal thickness, corneal thickness, and rete length were increased in psoriasis patients compared to controls. Dermal papillae tended to be narrower in patients than in controls, but the difference was not statistically significant, and suprapapillary epidermal thickness was similar in the two groups. Similarly, when the patients with plaque type psoriasis were compared with the control group, epidermal thickness, corneal thickness, rete length, and dermal papilla distance were significantly different, whereas there was no significant difference in suprapapillary epidermal thickness. The other types (inverse, erythrodermic) were not compared because of an insufficient number of patients.

Wang et al. (8) searched for a relationship between family history and severity of the disease in a total of 547 psoriasis cases and found no significant differences in the severity of the disease or in predisposing factors between the groups with and without a family history. In the study of Stuart et al. (9), in which the relationship between disease severity and a family history was examined in 537 psoriasis patients, analysis of familial psoriatics revealed that $\%$ TBSA was $15.1 \%$ when onset was early ( $\leq 40$ years of age), but only $8.7 \%$ when onset was late. The opposite trend was observed when psoriasis was sporadic; \% TBSA was $14.3 \%$ when onset was early, compared to $28.0 \%$ when onset was late. Rahman et al. (10) reported that there were more actively inflamed joints in nonfamilial psoriasis patients. Although it has been reported that family history has no effect on disease severity, some investigators have stated that \% TBSA is lower in lateonset cases. In the present study, rete length was $27 \%$ greater in psoriasis patients without a family history than in patients with a family history. The difference in rete length between patients with and without a family history was significant $(t=-3.334, P=0.03)$. The differences in full epidermal thickness, stratum corneum thickness, suprapapillary epidermal thickness or dermal papillary distances were not statistically significant $(\mathrm{P}>0.05)$. 
The corneal thickness of patients without scalp involvement was $75 \%$ greater than in patients with scalp involvement. The difference in corneal thickness between patients with and without scalp involvement was significant $(\mathrm{t}=-2.651, \mathrm{P}=0.008)$. The differences in epidermal, rete and suprapapillary epidermal thickness, and dermal papillary distance were not statistically significant between patients with and without scalp involvement ( $\mathrm{P}>0.05)$. Kaur et al. (11) observed that the scalp was the first site involved in $25.2 \%$ of patients, and stated that whether the fact that the corneum was thinner in psoriasis patients with scalp involvement was a definitive factor for dose adjustment in treatment should be studied further.

The duration of disease, \% TBSA and nail involvement caused no significant change in epithelial thickness in psoriasis patients. In the study by Stuart et al. (9), nail involvement was more common in earlyonset psoriasis; however, there were no dif- ferences between familial and nonfamilial cases. When the results concerning the biopsy sites were compared between patients and controls, the most prominent increase in full epidermal thickness and corneal thickness was observed in the palmar region and the least increase in the elbows of patients. Also, suprapapillary epidermal thickness was prominent in the palmar region but not in the elbows. The difference between dermal papilla widths was quite small.

In conclusion, epithelial thickness can be sensitively measured in skin biopsies using computer-supported image analysis. Parameters that involve epithelial characteristics, such as stratum corneum thickening or rete elongation, can be determined objectively. Since the follow-up periods and the number of patients in the present study were not sufficient, the relationship between epithelial thickness observed during the follow-up period and the response to various treatment modalities was not analyzed.

\section{References}

1. Ormerod AD, Dwyer CM, Weller R, Cox DH \& Price R (1997). A comparison of subjective and objective measures of reduction of psoriasis with the use of ultrasound, reflectance colorimetry, computerized video image analysis, and nitric oxide production. Journal of the American Academy of Dermatology, 37: 51-57.

2. Bacharach-Buhles M, el Gammal S, Panz B \& Altmeyer P (1994). The pseudo-elongation of capillaries in psoriatic plaques. Acta Dermato-Venereologica, 186 (Suppl): 133-137.

3. Kim SD, Huh CH, Seo KI, Suh DH \& Youn JI (2002). Evaluation of skin surface hydration in Korean psoriasis patients: a possible factor influencing psoriasis. Clinical and Experimental Dermatology, 27: 147-152

4. Ring J, Kowalzick L, Christophers E, Schill WB, Schopf E, Stander M, Wolff HH \& Altmeyer P (2001). Calcitriol 3 microg g-1 ointment in combination with ultraviolet $\mathrm{B}$ phototherapy for the treatment of plaque psoriasis: results of a comparative study. British Journal of Dermatology, 144: 495-499.

5. Ashcroft DM, Wan Po AL, Williams HC \& Griffiths CE (1999). Clinical measures of disease severity and outcome in psoriasis: a critical appraisal of their quality. British Journal of Dermatology, 141: 185-191.
6. Tanaka M, Gaskell S, Edwards C \& Marks R (2000). Simple horizontal averaging programme enables shade correction for image analysis in psoriasis. Clinical and Experimental Dermatology, 25: 323326.

7. Suh DH, Kwon TE, Kim SD, Park SB, Kwon OS, Eun HC \& Youn JI (2001). Changes of skin blood flow and color on lesional and control sites during PUVA therapy for psoriasis. Journal of the American Academy of Dermatology, 44: 987-994.

8. Wang $H$, Zhang $X \&$ Yang $S$ (2001). Study on the risk factors of psoriasis. Zhonghua Liuxingbingxue Zazhi, 22: 215-218.

9. Stuart P, Malick F, Nair RP, Henseler T, Lim HW, Jenisch S, Voorhees J, Christophers E \& Elder JT (2002). Analysis of phenotypic variation in psoriasis as a function of age at onset and family history. Archives of Dermatological Research, 294: 207-213.

10. Rahman P, Schentag CT, Beaton M \& Gladman DD (2000). Comparison of clinical and immunogenetic features in familial versus sporadic psoriatic arthritis. Clinical and Experimental Rheumatology, 18: 7-12.

11. Kaur I, Handa S \& Kumar B (1997). Natural history of psoriasis: a study from the Indian subcontinent. Journal of Dermatology, 24: 230-234. 